\title{
A Location-aware Guide based on Active RFIDs in Multi-Device Environments
}

\author{
Giuseppe Ghiani, Fabio Paternò, Carmen Santoro, Lucio Davide Spano \\ ISTI-CNR, Via G.Moruzzi,1 \\ 56124, Pisa, Italy \\ \{Giuseppe.Ghiani, Fabio.Paterno, Carmen.Santoro, Lucio.Davide.Spano\}@isti.cnr.it
}

\begin{abstract}
In this paper, we propose a multi-device, location-aware museum guide. It is a mobile guide able to opportunistically exploit large screens when they are nearby the user. Various types of games, which can exploit multi-device environments, are included in addition to the museum and artwork descriptions. The mobile guide is equipped with an RFID reader, which provides information useful to automatically detect nearby artworks. We also present example applications of this solution and then briefly discuss the results of first empirical tests performed to evaluate the usefulness and the usability of the enhanced mobile guide.
\end{abstract}

Keywords: Mobile Guides, Multi-device Adaptation, Location-awareness, RFIDs.

\section{Introduction}

Recent technological advances (including increasing availability of various types of interactive devices, sensors and communication technology) enable novel interactive software environments to support users in several contexts for different objectives. In the edutainment area, museums are an interesting domain because of the large amount of digital information, and the increasingly prevalent technological resources adopted in such environments. Thus, they are a particularly suitable context in which to experiment with new interaction techniques for guiding mobile users and improving their experience. However, at the same time, such a wealth of both information and devices might become a potential source of disorientation for users, if not adequately supported. 
Traditionally, the support for museum visits is limited to audio guides and interactive kiosks, which are limited in different ways from various viewpoints. It is important to exploit new technologies to identify new solutions able to enhance the user experience. Games for mobile guides can provide an interesting and amusing way to promote user interaction and learning. In this regard, the use of different types of devices within the museum (e.g.: mobile devices and large screens) can be seen as a means to enrich user experience by enabling further functionality, such as displaying the visitors' position and supporting games on the large screen while the user visits the museum using the mobile guide.

For lack of space we cannot discuss the many proposals put forward in the area of mobile guides. One important example was the GUIDE project (Cheverst et al., 2000), which developed an intelligent and context-aware tourist guide: each point of interest is associated with a geographic area, each one supported by a Wireless LAN, and when the user enters these areas the corresponding information is automatically provided as text descriptions, images and audio comments. Among other examples of mobile guides that exploit various kinds of information to adapt the services they provide, we mention Hippie (Opperman et al., 1999), which provides the visitors of an art exhibition with comments on the artworks in sight, adapting the information to user's location, interests and knowledge that is derived from the interaction. However, solutions based on automatic generation of comments on the closest artwork may sometimes be judged annoying by the user. CRUMPET (Poslad et al., 2001) personalizes its services, both on PDAs and mobile phones, not only according to the position of the user and her interests, but also according to previous interaction with the system. Lol@ (Pospischil et al., 2003) is a tourist guide for the city of Wien, using GPS as localisation techniques and a GIS support for generating the maps. It adapts the information to the device, but not to the user's characteristics. However, differently from our approach no multi-device support has been deployed in such guides.

We found interesting the idea of developing games to enhance interactivity in museums, which has been extensively explored mainly through more conventional media such as paper-based treasure hunts. However, little research has been reported in the role of digital adaptive games inside galleries, even if similar research in other contexts has been carried out (Bell et al., 2006). Furthermore, an increasing interest has been attracted by the use of RFID technology in order to supplement physical artwork with associated digital information. In (Mantyiarvi et al., 2006) we proposed the scan and tilt interaction paradigm. Physical selection is obtained by scanning RFID tags associated with the artworks and single handed tilt gestures are used to control and navigate the user interface and multimedia information. By pointing at the artwork of interest and controlling audio information with small single hand gestures, the visual channel is not too overloaded, resulting in a less intrusive interaction technique. We performed a first empirical evaluation on this prototype. The test showed an overall good acceptance among users but, at the same time, highlighted some limitations. For example, the passive RFID tags 
used in this prototype forced the users to stand in very close proximity to the artworks, which is not very natural in museum environments.

Here we present a new environment (UbiCicero) aiming to support multidevice interaction and games to improve museum visitor learning integrated with a location-aware support exploiting RFID technology. The goal is to improve the experience of museum visitors facilitating the access to the information available and increasing the interactivity of the environment.

In the paper, we first discuss related work, including our previous experiences with mobile guides; then, we present our novel approach for mobile guides exploiting in an integrated manner RFID technology, games and large shared screens and report on some first evaluations. Lastly, we provide some concluding remarks and indications for future work.

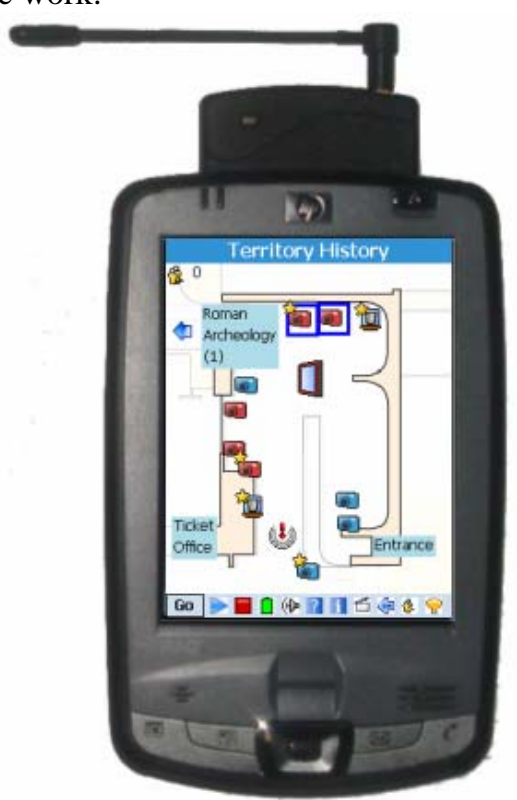

Figure 1. The Museum Mobile Guide

\section{The Visit}

Our interactive environment for museum visitors has been implemented for two museums: The Marble Museum (Carrara) and the Museum of Natural History (Calci). Our mobile guide provides visitors with a rich variety of multimedia (graphical, video, audio, ...) information regarding the available artworks and related items. In addition to information regarding artworks, sections and the museum, the application is able to support some services such as showing the itinerary to get to a specific artwork from the current location. Most information is 
provided mainly vocally in order to allow visitors to freely look around, however, the visual interface can show related videos, maps at different levels (museum, sections, rooms) and specific pieces of information.

One feature in the guide is the use of active RFID tags for automatically detecting nearby artworks. Figure 1 shows the museum guide implemented on the PDA device equipped with the Compact Flash RFID reader, which is a small add-on that can be plugged into a standard PDA interface. RFID-based solutions are composed of two main parts: the set of tags, or radio transponders and the tag reader, or transceiver. Tags basically have a static identification number (ID), but can also store different type of information such as sensed data (e.g., environmental temperature). The reader scans for available tags and, depending on their features, may interrogate them for additional information stored on their embedded memory. To make our mobile guide as small and as light as possible, we opted for a totally handheld-based solution consisting of Compact Flash (CF) RFID reader with small-sized antenna. The PDA does not need any additional expansion or adapter because the reader plugs directly into the CF slot. In general, RFID technology can be applied by using passive or active tags:

- Passive RFID tags do not have any internal battery and exploit the energy electromagnetically inducted by a neighbouring antenna. Thus, passive tags can respond with their ID when and only when a reader within a few centimetres' range interrogates them.

- Active RFID tags are equipped with an internal power source and are able to transmit by themselves at any time. There are two different types of active tags, depending on the way they work: beacon and respond.

o Beacon tags have a radio transmitter and they continuously send their data at certain intervals of time. The reader tunes into the proper radio frequency and listens to the tag information and the detection is not affected by the number of users, since the readers do not send any request to the tags. Also, beacon technology uses read-only tags

o Respond tags (which, differently from the previous ones, are read and write tags) wait for a reader request before responding. Due to the need for both transmitter and receiver modules, response tags involve more complex architectures, larger dimensions and higher costs than beacon tags. However, while the battery life of broadcast tags (2-4 years) depends on how frequently they transmit, the battery life of respond tags depends on how often they are interrogated (in any case more than 6 years).

An undeniable benefit of active tag technology is the wide read range (up to 100 meters). Museum artworks are equipped with physical tags and each tag has a unique ID number. Note that a single tag can be associated with more than one neighbouring artwork, when they are very close. This is due to the difficulty of distinguishing two or more tags that are too close to each other. Indeed, if two tags were placed in a very small area, the reader would see both of them with the same RSSI (Received Signal Strength Indication). The use of RFID technology for lo- 
calization and the problems related to tag density are also discussed in (Bellotti et al., 2006). When the user enters a new room, the guide activates the museum map highlighting the new room and corresponding vocal comments.

Continuous monitoring of the tags' signal allows the guide to calculate artworks closest to the user among those inside the current room. When a new tag is detected, i.e. a new area is being visited, an audio clip is played to alert the user and a vocal message indicating the number of neighbouring artworks is generated. Tag detection enables the computation of user's position; however, the proximity of an artwork does not imply the user is interested on that artwork (s/he could be even looking to another one). For this reason, and depending on user preferences, the guide may ask confirmation before describing any artwork or even describe them automatically. A more affordable support would detect user's orientation through an electronic compass (see section 6) besides user location to determine which item s/he is actually watching at.

We chose the active beacon tags for our RFID-based solution because ti is more scalable with respect to the number of users. Actually, the RFID reader just tunes into the proper radio frequency and "listens" to the tag(s) information, reporting the list of visible tags together with their RSSI (Received Signal Strength Indication). However, when tags are sending their IDs, overlap may occur and data may be lost. For this reason, the detection of all the visible tags may take a few seconds (depending on the number of tags in range).

Localization at the application layer is performed by estimating the nearest tag and obtaining the associated artwork(s). Access to the RFID hardware is achieved via the libraries provided by the hardware supplier (http://www.identecsolutions.com/) whose functions allow the application to configure and interrogate the RFID reader. For each query (queries are performed with the frequency of two per second) from the localization support the RFID reader provides the list of visible tags. Each list element contains data related to a visible tag, such as ID and RSSI. The application localization support keeps a list of all the tags that have been detected at least once. In the application each tag is associated with its last reported RSSI and the time elapsed since its last detection. Only tags that have been detected recently are considered in the computation. The best tag is always the one with the highest RSSI. However, a "new tag event" is generated only if the new tag is the best candidate for $\mathrm{N}$ consecutive queries. The value of $\mathrm{N}$ is specified in the application settings to achieve a trade-off between reliability and speed of the localization: the higher it is, the more reliable the localization will be, but also the more time it will take to update the identification of the closest artworks. The value of $\mathrm{N}$ must be carefully chosen, especially when tag density is high (i.e. artworks are very close), in order to avoid erroneous detections. To facilitate localization an RSSI threshold is used to adjust the reader sensitivity. In our case, we are able to detect tags up to five meters, also because the limitations of the small antenna. Lower sensitivity makes the reader report only the nearest tags and simplifies the application layer computation. Nevertheless, when many artworks are too close to each other they may be detected as a single 
tag. Indeed, if two tags were placed in a very small area, the reader would see both of them with the same RSSI. The guide automatically detects this movement through the RFIDs and highlights nearby artworks with squared frames around the corresponding icons. Then, when the user accesses the description of the artworks the colour of the associated icons changes again to signal the fact that the related artworks have been visited.

\section{Software Architecture}

The main elements of the software architecture are the modules in the PDA, in the stationary device and the communication protocol of the environment. The PDA module is composed of five layers, each one provides the others with services. From the bottom they are:

- Museum DB, which provides information about the artworks

- Core and Communication, which provides the basic mechanisms, including network services for sending/receiving messages;

- Localization, which provides support for localizing the nearby artworks and the user location exploiting various technologies (such as RFID and IrDA), when available;

- Visit, supporting interactive access to museum info;

- Games, supporting the interactive games

In particular, the database provides information about museum artworks to the upper layers.

The core implements data structures useful for the upper layers, e.g. support for configuration and help and the XML parsers. It also provides functionality used to update the information regarding the state of the players, to connect to shared stationary displays and to exchange information among palmtops and therefore implements algorithms for managing sockets, messages and group organisation. When the user explicitly starts the interface splitting, the mobile application sends a connection request message to the stationary device. If the connection is successful, a precomputed desktop version of the application is activated, a series of messages encoding the state data is sent by the mobile version to the stationary one to replicate the state. State synchronization must be maintained when the interface is split between the mobile and the situated display. Thus, the mobile has to send update messages anytime its state changes.

The localization layer contains the concurrency manager of IrDA and RFID signals (infrared signals are used to detect when the user enters a room). Such layer is exploited by the visit and game layers. The visit layer supports the presentation of the current room map and a set of interactive elements. Each artwork is associated with an icon identifying its type (sculpture, painting, picture, ...) and positioned in the map according to its physical location. By selecting such icon, 
users can receive detailed information on the corresponding artwork. In addition, this part of the application allows users to receive help, access videos, change audio parameters and obtain other info.

The games layer extends the museum visit application and supports games displaying and solving. Game contents are defined using an XML format to allow easy modifications and additions.

\section{Visit and Games in Multi-Device Environments}

While some preliminary ideas regarding the use of games in mobile guides were introduced in (Dini et al., 2007) but without using any localization support, here we present a comprehensive solution, tested into two museums, with a wider set of games. Such games have been integrated with a RFID-based localization support. In particular, in our new environment in order to increase the learning experience six types of individual games are considered :

- In the associations game users must associate images with words, e.g. the author of an artwork, or the material of an artwork.

- In the details game an enlargement of a small detail of an image is shown. The player has to guess which of the artwork images the detail belongs to.

- The chronology game requires the user to arrange the images of the artworks shown chronologically according to the creation date.

- In the hidden word game, the users have to guess a word: the number of characters composing the word is shown as help.

- In the memory game, the user has to look at an image (which then disappears) and s/he has to answer to a question associated with the previously shown image.

- The quiz is a single-choice question.

The artworks that have an associated game show an additional star icon, through which it is possible to access the game. If the game is solved correctly the icon becomes a green check, otherwise it turns to a red cross (Figure 2).

The main feature of our solution is to support visit and game applications exploiting both mobile and stationary devices equipped with large screens. The typical scenario is users freely moving and interacting through the mobile device, who can also exploit a larger, shared screen of a stationary device (which can be considered a situated display) when it is nearby. Shared screens connected to stationary systems can increase social interaction and improve user experience, otherwise limited to individual interaction with a mobile device. They also stimulate social interaction and communication with other visitors, though they may not know each other. 
A larger shared screen extends the functionality of a mobile application enabling the possibility to present individual games differently, share social game representations, show the positions of the other players in the group and also perform a virtual pre-visit of the entire museum. Each large shared display can be in different states:

- Standalone: the screen has its own input devices (keyboard and mouse) and it may be used for a virtual visit of the museum. It can be exploited by visitors who do not have the PDA guide .

- Split: one visitor has taken control of the display. The PDA displays name and group of the controller.

- Search: the display shows the current position of the other players, according to the RFID localization.

- Game: the display shows one individual game.

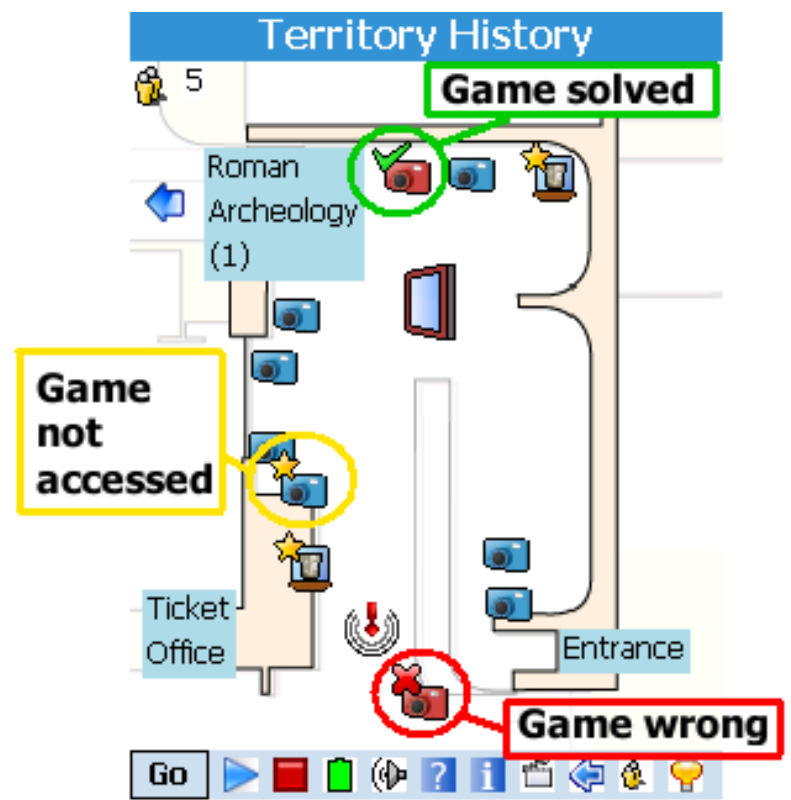

Figure 2: Representation of the game state.

Since a shared display has to go through several states the structure of its layout and some parts of the interface remain unchanged in order to avoid disorientating users. This permanent part of the user interface provides information such as the current section map and its position in the museum, an explanation of the icons used to represent the artworks and the state of the final enigma game. In standalone mode users can select from three kinds of views of the section map, using the toolbar on its top-left corner. These views are:

1. Icons: the artworks are represented by an icon indicating the type. 
2. Thumbnails: the icons are replaced by a small photo of the artwork

3. Thumbnails and icons: small artwork photos are accompanied by icons on their bottom-left corner (see Figure 3).

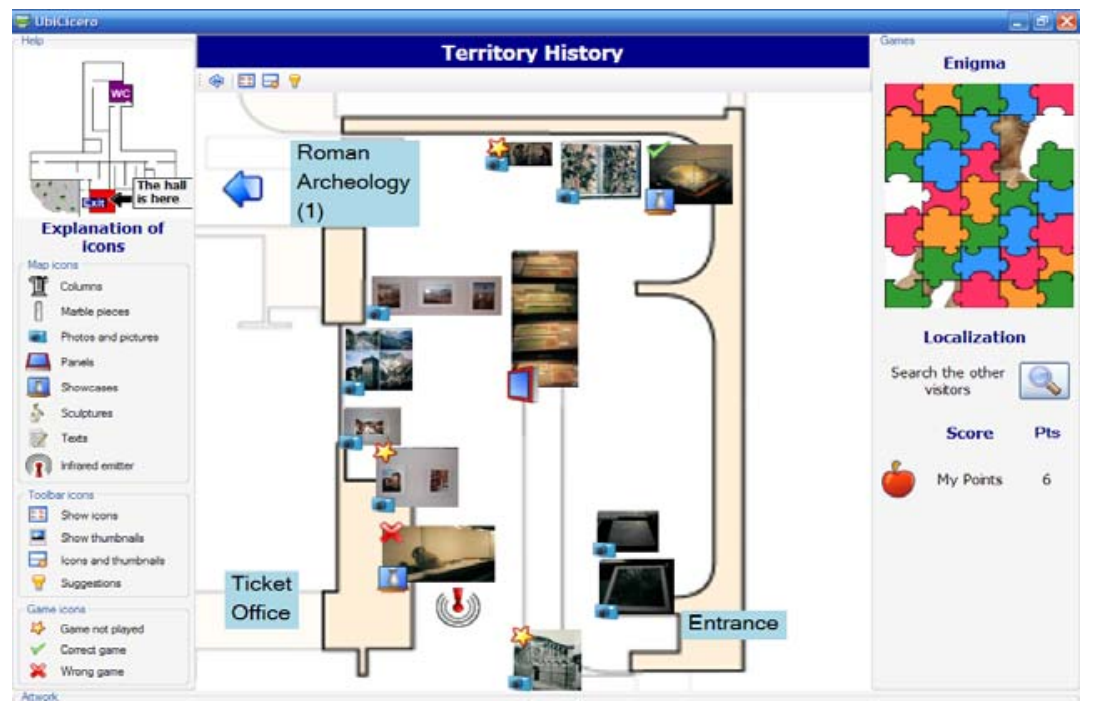

Figure 3: Museum room in large screen using thumbnails plus icons.

When an artwork or a game is selected, the screen interface changes its layout adapting the focus: it magnifies the correspondent panel and shows the artwork details (see Figure 4) or the game interface. It also shows the path to reach the artwork from the current position.

The screen changes its state to split when a player selects the connection through the PDA interface. In this case the large screen is used both to show additional information and also allow multiple users to focus the attention on a given game exploiting the screen size. When a player is connected to the large screen, its section map view is automatically changed to thumbnails, while the artworks types are shown on the PDA screen. The artwork presentation uses a higher resolution image on the large display, adding more information to the description. The game representation on the large screen is used to share and discuss it among multiple users. In the split representation, the possible game answer choices are shown only on the PDA interface, while game information and higher resolution images are shown on the larger screen (see Figure 5).

The visualisation of the enigma game differs depending on the availability of the large screen: if only the PDA is used, it is composed of two presentations visualised sequentially on the PDA, the hidden image and the associated questions. If the larger shared screen is available, then the hidden image is shown on the large display, while the answers are presented on the PDA user interface. 


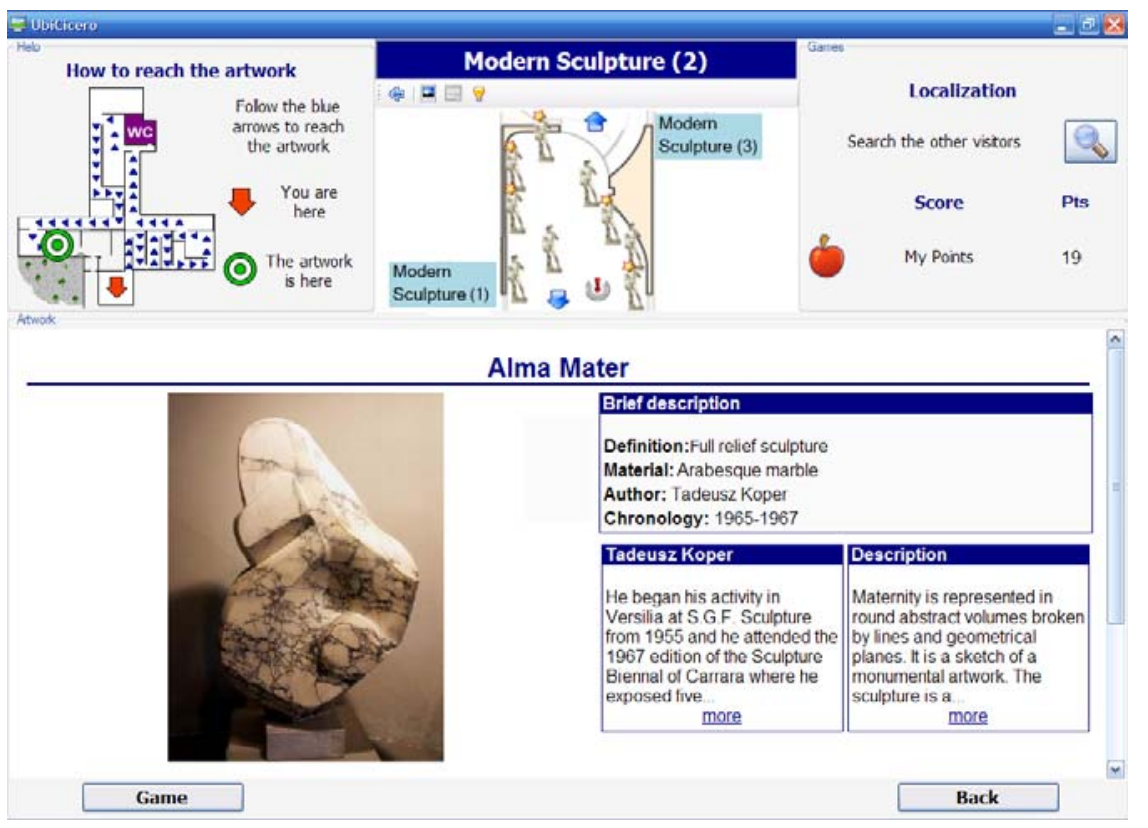

Figure 4: Artwork presentation on large screen.

Providing an effective representation of visitors' position on the PDA is very difficult because of the small screen, especially when they are in different rooms. In the case of the large shared screen it is possible to obtain a better representation, which is divided into sections, one for each visitor currently visiting the museum with a PDA equipped with RFID reader. Each part shows the name and the room where the visitor is located and the artworks close to the player are highlighted by rectangles.

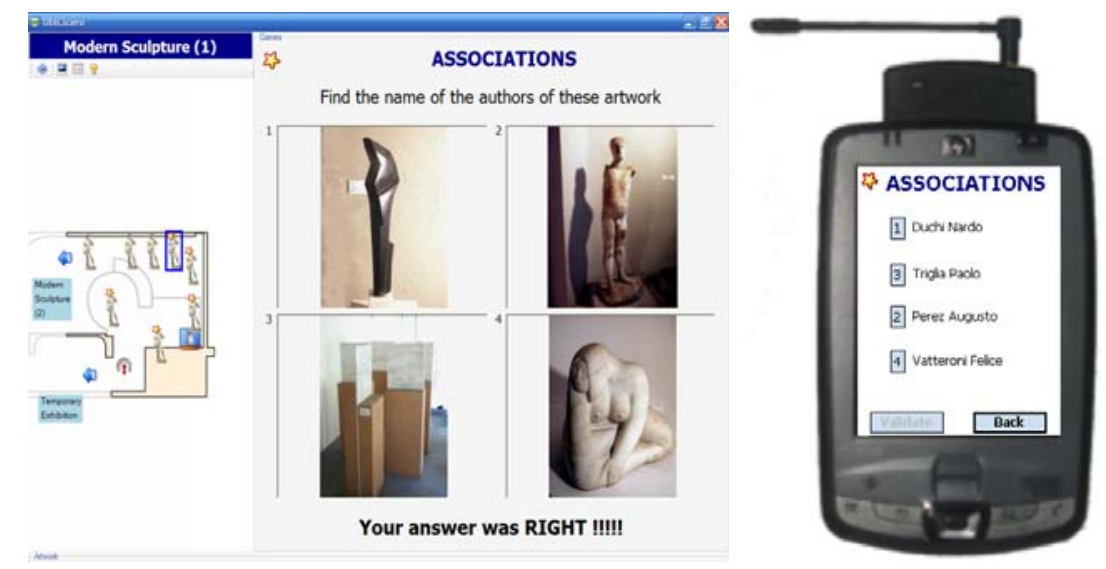

Figure 5: Example of game in distributed mode. 


\section{Evaluation}

We performed a first evaluation of our nomadic application guide involving 7 users in the Marble Museum and 5 in the Natural History Museum (8 males and 4 females), with an average age of 36,4 years old; 3 of them with secondary school education, the others with laurea degree. Users were requested to read a short introduction about the application and they were also instructed about the tasks they were expected to carry out. They were invited to test two versions of the mobile guide. One version was equipped with the multi-device support, the RFID module for detecting proximity of artworks within a museum room. The other version was a basic one without the multi-device support and no RFID support.

They were asked to visit some sections of the museum using the two versions. With the enhanced prototype they were also requested to access and solve some games and to perform at least one splitting between PDA and a large screen available in a museum section. Half of the users started the visit by using the enhanced version and then continue with the basic one; for the others the vice versa held. Afterwards, the users had to fill in a questionnaire.

Almost all people involved in the tests reported not to have good experience in using PDAs, a few of them had previous experience in using digital museum guides, even less reported to have used digital games in museum settings.

The possibility of the enhanced museum guide of detecting the current position of the user and therefore to present information dependent on such a position (by surrounding with a frame the icon representing the artwork(s) closest to the user) was judged useful by testers (in a 1-5 scale in which 1 is the worst case and 5 is the best one, $\mathrm{M}=3,58 ; \mathrm{SD}=1,16$ ) especially for orientation and for helping in identifying an artwork and associating an icon in the map to the related artwork in the museum. Delays in the localisation support were noticed by some users, which brought about some hesitations during the visit. In addition, they judged useful $(M=3,75 ; S D=0,87)$ the support given by the enhanced guide for presenting descriptive information about the artworks currently closer to the user. As for the multi-device support, users appreciated the guide/games support offered in the PDA version $(M=3,73)$ and in the desktop one $(M=3,2)$. Also the way in which the functionality was split between the PDA and the large screen was rated quite positively $(\mathrm{M}=3,1)$. Regarding the splitting, some of them noted that it might be not so easy for the user to follow a description which is partially visualised on a PDA and partially on a large screen because of the division of attention.

People who preferred the visit with the basic prototype appreciated the possibility of having a quicker visit and the fact that the basic version supported a visit that allows more user's initiative with respect to the enhanced version. The UI for visualising the games associated to the various artworks in the museum was rated quite good $(M=3,67 ; S D=1,37)$, although some users reported problems in selecting the icon representing the game associated to a specific artwork; regarding the content of the games, users judged them in a quite good way $(\mathrm{M}=3,58$; $\mathrm{SD}=$ 
1,24), although they emphasised the opportunity of providing games that are easy for being solved by museum visitors. Games were judged amusing $(\mathrm{M}=3,67$; $\mathrm{SD}=$ $1,15)$ and useful in stimulating and improving learning $(\mathrm{M}=3,5 ; \mathrm{SD}=1,31)$, one of the most favourite games was the quiz.

All in all, the application was acknowledged useful, interesting and with good potentialities. Improvements were suggested in order to further improve the precision of user localisation within the museum sections and its performance in the location support and to simplify as much as possible the interaction.

\section{Conclusions and Future Work}

In this paper, we proposed a multi-device, location-aware guide supporting museum visits, including the possibility of enriching the museum visits through games. Its main contribution is in the ability to exploit multi-device environments, in which users can freely move about with their mobile guide but also exploit large screens connected to stationary PCs when they are nearby. Both the access to museum information and the associated games can benefit from the availability of multiple devices as well as additional services, such as the presentation on the large screen of the locations of other visitors, which are detected through RFID tags. We have also developed an authoring environment which allows developers to easily customize the guide for new museums.

We are investigating new ways to further enhance personalisation of the guide user interface, in particular in group visits, and the possible automatic detection of user's orientation. Orientation, combined with localization, would better support the identification of the user's interests in the exhibition area.

Future work will be dedicated to carry out further empirical validation of our guide in order to better understand the advantages and possible improvements of the enhanced version with respect to the basic one.

\section{References}

Bell, M., and M. Chalmers, L. Barkhuus, M. Hall, S. Sherwood, P. Tennent, B. Brown, D. Rowland, S. Benford, M. Capra, A. Hampshire: 2006, Interweaving Mobile Games With Everyday Life. CHI 2006 Proceedings Games and Performances, Montréal, Canada.

Bellotti, F., Berta, R., De Gloria, A., Margarone, M. Guiding visually impaired people in the exhibition. Mobile Guide 2006, 2006 (http://mobileguide06.di.unito.it/pdf/Bellotti\&al.pdf).

Cheverst, K., and N. Davies, K. Mitchel, P. Smyth: 2000, Providing tailored context-aware information to city visitors. In Adaptive Hypermedia and Adaptive Web-Based Systems (AH 2000). LNCS Vol. 1892, Springer-Verlag, Berlin Heidelberg New York, pp. 73-85 
Dini R., Paternò F., Santoro C., An Environment to Support Multi-User Interaction and Cooperation for Improving Museum Visits through Games, Proceedings Mobile HCI’07, Singapore, September 2007, ACM Press.

.Mantyiarvi, F.Paternò, Z.Salvador, C.Santoro, Scan and Tilt - Towards Natural Interaction for Mobile Museum Guides, Proceedings Mobile HCI 2006, pp.191-194, ACM Press, Espoo, September 2006..

Opperman R., and M. Specht, I. Jaceniak: 1999, Hippie: a nomadic information system. Handheld and Ubiquitous Computing. Springer-Verlag, Berlin, pp. 330-333.

Poslad, S., Laamanen, H., Malaka, R., Nick, A., Buckle, P., Zipf, A.: CRUMPET: Creation of User-Friendly Mobile Services Personalised for Tourism. 3G Mobile Communication Technologies. London, UK (2001) 26-29

Pospischil, G., and M. Umlauft, E. Michlmayr: 2003, Designing LoL@, a Mobile Tourist Guide for UMTS. Information Technology \& Tourism 5(3), Cognizant Communication Corporation, pp. 151-164. 